\title{
Publisher's Note: Analytical description of photon beam phase spaces in inverse Compton scattering sources [Phys. Rev. Accel. Beams 20, 080701 (2017)]
}

C. Curatolo, I. Drebot, V. Petrillo, and L. Serafini (Received 20 September 2017; published 3 October 2017)

DOI: 10.1103/PhysRevAccelBeams.20.109901

This paper was published online on 3 August 2017 with an error in Eq. (20). Equation (20) should read as

$$
\mathcal{N}^{\Psi}=6.25 \times 10^{8} \frac{U_{L}(\mathrm{~J}) Q(\mathrm{pC}) r}{E_{L}(\mathrm{eV})\left[\left(\sigma_{x}^{2}(\mu \mathrm{m})+\sigma_{L}^{2}(\mu \mathrm{m})\right]\right.} \frac{\left(1+\sqrt[3]{X} \Psi^{2} / 3\right) \Psi^{2}}{\left[1+(1+X / 2) \Psi^{2}\right]\left(1+\Psi^{2}\right)}
$$

The equation has been corrected online as of 14 September 2017. 